\title{
ANALYSIS OF CD80- AND CD86-EXPRESSING B-LYMPHOCYTE LEVELS IN THE BLOOD OF WOMEN WITH LOCALLY ADVANCED TRIPLE- NEGATIVE BREAST CANCER
}

Amanda Forte Mendes Tejo Salgado1,2,3, Maria Eduarda Borges Kerstenetzky ${ }^{1,2,3}$, Vitoria Ferreira David Melquiades ${ }^{1,2,3}$, Marcelo Ramos Tejo Salgado4, Leuridan Cavalcante Torres ${ }^{1,2}$

${ }^{1}$ Instituto de Medicina Integral Professor Fernando Figueira - Recife (PE), Brazil.

${ }^{2}$ Translational Research Laboratory Prof CA, Hart, Instituto de Medicina Integral Professor Fernando Figueira - Recife (PE), Brazil.

${ }^{3}$ Faculdade Pernambucana de Saúde - Recife (PE), Brazil.

${ }^{4}$ Hospital de Câncer de Pernambuco - Recife (PE), Brazil.

Objective: To assess the levels of CD80 and CD86-expressing B lymphocytes in the blood of women with locally advanced triple-negative breast cancer (TNBC). Methods: This is a prospective and exploratory cohort study involving 30 women with TNBC and 30 healthy controls, conducted in 2018-2019. Peripheral blood collection was performed prior to chemotherapy. Immunophenotyping of B lymphocytes and CD80 and CD86 molecules was performed by flow cytometry. Women were evaluated for the degree of pathological response to chemotherapy and divided into groups with full (RC) or partial (RP) pathological response. Nonparametric Mann-Whitney U-test was used for comparison between two groups. Values of $\mathrm{p}<0.05$ were considered statistically significant. Analyses were performed using Graphpad v7.0 software. Results: We analyzed 30 patients with locally advanced TNBC. The age of the patients ranged from 27 to 59 years, and median age was 44.5 years (35.5-51.7). Regarding menopausal status, $62.1 \%$ were premenopausal and $37.9 \%$ postmenopausal. Regarding the nuclear grade, $63 \%$ of the tumors were grade 3, followed by $27 \%$ grade 2 . In relation to the clinical stage, $30 \%$ were in stage IIIA, 63.4\% stage IIIB, and 6.6\% stage IIIC. In the evaluation of response to neoadjuvant treatment, $56.7 \%$ of patients had complete pathological response, and $43.3 \%$ had partial response. TNBC patients had high levels of CD86 + B lymphocytes when compared with controls ( $\mathrm{p}<0.0001)$. Regarding total B and CD80 + B levels-no significant differences were observed between the groups. In the analysis of CD86 and CD80 expression and total B cell levels, no significant differences were observed between the $\mathrm{RC}$ and $\mathrm{RP}$ groups. Conclusion: This study showed that the immune system of patients with TNBC can regulate costimulatory molecules in circulating B cells, probably in response to the disease.

Keywords: Breast Neoplasms; Neoadjuvant Therapy; CD80/86, B7-1/2. 